\title{
Seeking Tutorial Articles for Attention, Perception, \& Psychophysics
}

As I hope you have noticed, $A P \& P$ has begun publishing a series of tutorial reviews. Perhaps you are just the person to write one. Tutorial reviews are written at the invitation of the editor or an associate editor, but I invite you to suggest yourself for an invitation. Look at the guidelines and contemplate the possibility that you should write a tutorial.

The goal. We aim to create a series of articles that can be first stops for anyone wanting to get up to date on a topic. They would be perfect for a grad student who wanted an introduction to a topic or, perhaps, for a principal investigator whose grant reviews suggest learning about a topic. An undergrad with no background might find these tutorials heavy going, and an expert on the topic would not look here for an extended argument about the latest arcane points of controversy.

Scope. Broad, but not too broad. "Attention" or "binocular vision" would be far too broad. "Visual search for binocular stimuli" would be too narrow. Scope can be discussed with one of the editors.

The author. You should have a recognized track record of research on the topic.

Length. Longer than in Current Directions in Psychological Science, shorter than in Annual Review of Psychology. The main text should be 5,000-8,000 words, excluding figures and bibliography.

Bibliography. Extensive. One of the goals is to have a comprehensive list of citations in one place. The citations should go well beyond the work of the author's lab.

Ideological purity. It would not be reasonable to ask authors to suppress their own theoretical positions. However, these tutorials should make an effort to be even-handed.

Figures. Encouraged.

Review. A tutorial article will be reviewed like a regular submission to $A P \& P$, but with the expectation that invited tutorials will eventually be accepted, assuming that the author responds to reviews in a reasonable manner.

Your next step. If you have an idea for an excellent tutorial, send a brief description (a paragraph or outline, nothing much longer) to wolfe@search.bwh.harvard.edu. I look forward to hearing from you.

Jeremy M. Wolfe Editor 